\title{
High Electrical Conductivity, Thermal Stability and Gas Sensing Studies on Silver-Polyaniline Nanocomposite
}

\author{
Sanjib Baglari ${ }^{1}$ and D. Sarkar ${ }^{2}$ \\ ${ }^{1,2}$ Department of Physics, Gauhati University, Guwahati, India \\ *Corresponding author Email: sanjibbaglari@gmail.com
}

Available online at: www.isroset.org

Received: 09/Mar/2019, Accepted: 04/Apr/2019, Online: 30/Apr/2019

\begin{abstract}
This paper reports the synthesis of Polyaniline-Silver (Pani/Ag) nanocomposites by an in-situ chemical polymerization method using ammonium persulphate (APS) as an oxidizing agent. The prepared Pani/Ag nanocomposite were characterized by XRD, EDAX, FESEM, TEM, TGA, UV-vis spectroscopy. The XRD patterns indicated that the crystalline phase of $\mathrm{Ag}$ is cubic with average crystallite size of $26 \mathrm{~nm}$. UV-vis spectroscopy analysis indicated that the Ag nanoparticles have some effect on the Pani matrix. The TGA result showed that thermal stability of the nanocomposite was increased after incorporation of Ag nanoparticles. Pani/Ag nanocomposite showed superior DC conductivity when compared to pure Pani. The Pani/Ag nanocomposite also showed excellent chloroform sensing behaviour than pure Pani, which might be due to increase in the surface area of Pani in the presence of Ag nanoparticles.
\end{abstract}

Keywords: Polyaniline, Nanocomposite, DC Conductivity, Gas Sensing, Thermal Stability, UV Vis.

\section{INTRODUCTION}

Conducting polymer exhibit both conducting and semi conducting properties. Recently, conducting polymers has received much attentions in research because of their promising optical properties, controllable chemical and electrochemical properties, easy processability, ease of synthesis, inexpensive monomer and environmental stability [1-4]. Among the conducting polymers, polyaniline (PANI) has been studied extensively worldwide for its various applications in the design of sensor [5-6], optoelectronics device [7], secondary batteries, fuel cell [8], super capacitor [9] and catalytic properties [10].

In recent years, composites of polyaniline with different metal oxide for gas sensing applications has received great attention worldwide because of its higher sensitivity, reversible response, quick response time and inexpensive monomer [11]. It has been reported that incorporation of metal nanoparticle to polyaniline exhibits excellent sensing properties with large effective surface area for chemical interaction and hence improve catalytic efficiency [12-13]. It was also reported that incorporation of metal nanoparticle effectively increased electrical, optical and dielectric properties of the polyaniline composite [14-15]. Satish Sharma et al [16] reported that the chemically synthesized Copper/Polyaniline nanocomposite gas sensor exhibited higher sensitivity, faster response time and recovery rates to chloroform vapour than those of a pure PANI sensor.
Mudassir Hasan et al [17] showed that PANI/Au nanocomposite exhibited a better ammonia sensing and recovery response than pure PANI. Athawale et al [18] demonstrated the potential application of the PANI/Pd nanocomposite as a selective methanol sensor. They found that sensor characteristics such as the response time and long-term stability of the PANI/Pd nanocomposite sensor were superior to pure PANI sensor. Therefore, it is hopeful to obtain PANI/Ag nanocomposites would be a novel material in the area of gas sensor with excellent gas sensing behaviour. To the best of our knowledge, the PANI/Ag nanocomposite as chloroform sensing materials have not been reported elsewhere. Chloroform is released into the environment through several anthropogenic sources where industrial sites using it in the production line. Exposure to 50 ppm of chloroform can damage human health [19]. Therefore, the reliable and quick detection of chloroform gas is needed.

The aim of this study was to prepare a Polyaniline/Ag nanocomposite for chloroform gas sensing. The combination of PANI with silver nanoparticle composite is expected to produce synergism between the constituents, which could effectively improve sensitivity and recovery behaviour for chloroform sensing application.

In this present work, PANI/Ag nanocomposites were prepared by an in-situ chemical polymerization method using APS as an oxidizing agent and hence explore the 
possibility of improving of conductivity and gas sensing property of Pani by doping it with silver nanoparticles.

\section{MATERIALS AND METHOD}

\section{A. Materials}

Aniline monomer, silver nitrate (AgNO3) and sodium borohydride $(\mathrm{NaBH} 4)$ were purchased from Sigma Aldrich (India) as analytical grade. APS, hydrochloric acid $(\mathrm{HCl})$ and Chloroform were obtained from Merck India Ltd. as reagent grade. All solutions of reacting materials were prepared in Deionized (DI) water.

\section{B. Synthesis of Ag nanoparticles}

Silver nanoparticles were synthesized by chemical reduction of AgNO3 using $\mathrm{NaBH} 4$ by a previously reported method [20]. In a typical experiment, Silver nitrate solution was separately prepared by adding different mole concentration, viz., $0.1,0.2$ and $0.3 \mathrm{M}$ of AgNO3 in DI water. The details of the method are as follows:

A $10 \mathrm{~mL}$ volume of $0.1 \mathrm{M}$ silver nitrate was added dropwise to $30 \mathrm{~mL}$ of $0.2 \mathrm{M}$ sodium borohydride solution under constant magnetic stirring at $0-5{ }^{0} \mathrm{C}$. The solution turned light yellow after the addition of $2 \mathrm{~mL}$ of silver nitrate and a brighter yellow when all the silver nitrate had been added. The clear yellow colloidal silver nanoparticle (Fig. 1) was stable at room temperature stored in a transparent vial for several weeks. The transmission electron microscopy (TEM) show that the average diameters of the $\mathrm{Ag}(0)$ particles were $\sim 10 \mathrm{~nm}$

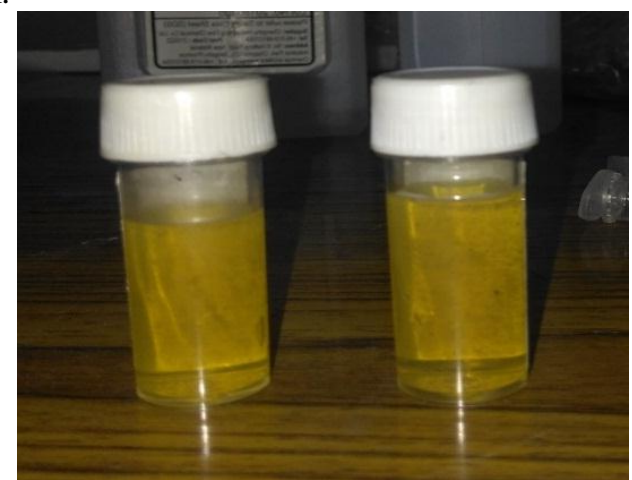

Figure 1. Silver Nano particle colloidal

\section{Synthesis of polyaniline-Ag nanocomposite}

The PANI/Ag nanocomposites were prepared by an in-situ chemical polymerization method using APS as an oxidizing agent in the presence of silver nanoparticles colloid. In a typical synthesis process, $0.2 \mathrm{M}$ Aniline is mixed with $1 \mathrm{M}$ HCL solution and then it is added to the previously prepared $40 \mathrm{~mL}(0.1,0.2,0.3 \mathrm{M})$ silver nanoparticles colloids under constant magnetic stirring for $2 \mathrm{~h}$. Finally, precooled aqueous solution of APS $(5.71 \mathrm{~g}, 25 \mathrm{mmol})$ is slowly added to the above mixture under constant magnetic stirring at 0 $5^{0} \mathrm{C}$ for next $6 \mathrm{~h}$. The colour of the solution transformed slowly to greenish black. The green solution was kept in the refrigerator for $24 \mathrm{~h}$ in order to complete the polymerization process. The resultant product was filtered with acetone, ethanol and DI water until the filtrated turned colourless. The pure PANI was prepared using same method as described above in the absence of silver colloid.

\section{Characterization techniques}

The phase identification and structural analysis of the powdered sample were carried out by using $X^{\prime}$ 'Pert pro $X$ ray diffractometer (XRD) in the range, $10-60^{\circ} 2 \theta$, using $C u K \alpha$ radiation $\left(\lambda=1.5406 A^{\circ}\right)$. The morphological and elemental analysis of the composites were carried out by field emission scanning electron microscopy (FESEM, ZEISS, SIGMA, Germany). The shape and size of silver nanoparticles were examined by HRTEM (JEM 2100, JEOL, Japan). Thermogravimetric analysis was performed from $25^{\circ} \mathrm{C}$ to $800^{\circ} \mathrm{C}$ at a heating rate of $10^{\circ} \mathrm{C} / \mathrm{min}$ in a nitrogen atmosphere using TGA (Model TGA-50, SHIMADJU. The UV-visible absorption spectroscopy was recorded by using CARY (Model 300) spectrophotometer (Australia) in the wavelength ranges from 200 to $850 \mathrm{~nm}$. The gas sensing measurement of pure PANI and PANI/Ag nanocomposite (as pellet) were performed at room temperature at different concentration of chloroform viz. 100, 200 and 300ppm. The sensing ability of the nanocomposite was tested through change in resistance in the sensor while exposed to chloroform vapours at room temperature. The sensitivity (S) of the sensor was calculated using the following equation.

$S=\frac{R_{\text {gas }}-R_{\text {air }}}{R_{\text {air }}}=\frac{\Delta R}{R_{\text {air }}}$

The DC electrical conductivity of the pellet was calculated using the following equation [21].

$$
\sigma=(\pi / \ln 2)(V / I) * t * F(t / s)
$$

where $\sigma, \mathrm{I}, \mathrm{V}, \mathrm{s}, \mathrm{t}$ and $\mathrm{F}(\mathrm{t} / \mathrm{s})$ are the $\mathrm{DC}$ electrical conductivity $\left(\mathrm{s} \mathrm{cm}^{-1}\right)$, current $(\mathrm{A})$, voltage $(\mathrm{V})$, probe spacing $(\mathrm{cm})$, thickness of the pellet $(0.1-0.3 \mathrm{~cm})$ and correction factor respectively. The change in resistance as a function of time (for gas sensing) and the voltage change for an injected current (for DC conductivity) were measured using a standard Keithley 2400 programmable source meter. All electrical contacts were made on the surface of the pellet with copper wire and conductive silver paste.

\section{RESULT AND DISCUSSION}

\section{A. Phase Identification Analysis}

The X-ray diffraction (XRD) patterns of pure PANI and PANI/Ag composite are shown in the Fig. 2 In this XRD pattern, the broad amorphous scattering peaks at $2 \theta \approx 25.3^{\circ}$ may arise due to regular repetition of monomer aniline. This can be attributed to the periodicity parallel and perpendicular to the polymer chains of PANI [22]. Moreover, it was also observed that intensity of the peak became stronger with 
increasing $\mathrm{Ag}$ concentration. This result indicated that incorporation of Ag to PANI improved the crystallinity of PANI. The prominent peaks at $2 \theta=38.3^{\circ}$ and $44.5^{\circ}$ to the (111) and (200) diffraction planes respectively, ascribed to the face cantered cubic (FCC) structure of Ag [23]. The average crystalline size (D) was calculated using the Scherrer formula [24]:

$$
D=\frac{0.94 \lambda}{\beta \cos \theta}
$$

Where $\lambda$ is the wavelength of $\mathrm{X}$-ray $\left(1.5406 A^{\circ}\right), \theta$ is the half diffraction angle of peak (in degrees) and $\beta$ is the full width of half maximum of intense peak. The average crystalline size calculated through the (111) plane is $\sim 26 \mathrm{~nm}$.

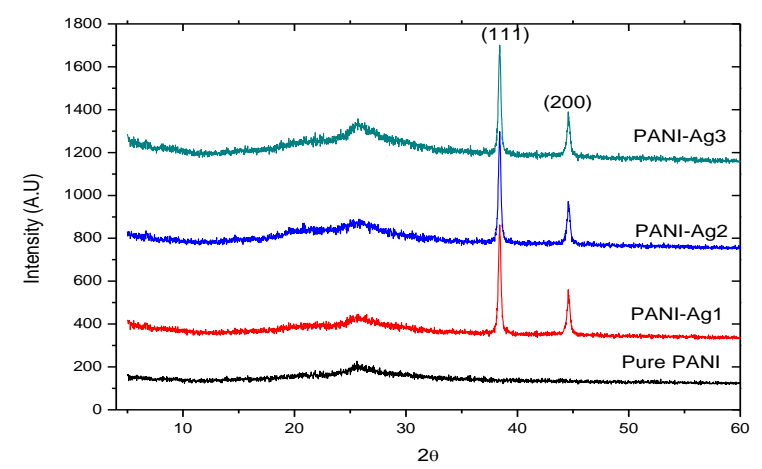

Figure 2. XRD pattern of PANI and PANI/Ag composite

\section{B. TEM analysis}

Fig. 3 shows TEM image of silver colloidal nanoparticle. The shape and size of the resultant particles were estimated with the help of TEM. The obtained image suggest that the morphology of the Ag nanoparticle was spherical in shape and the average size of $\mathrm{Ag}$ nanoparticle were around $10 \mathrm{~nm}$.

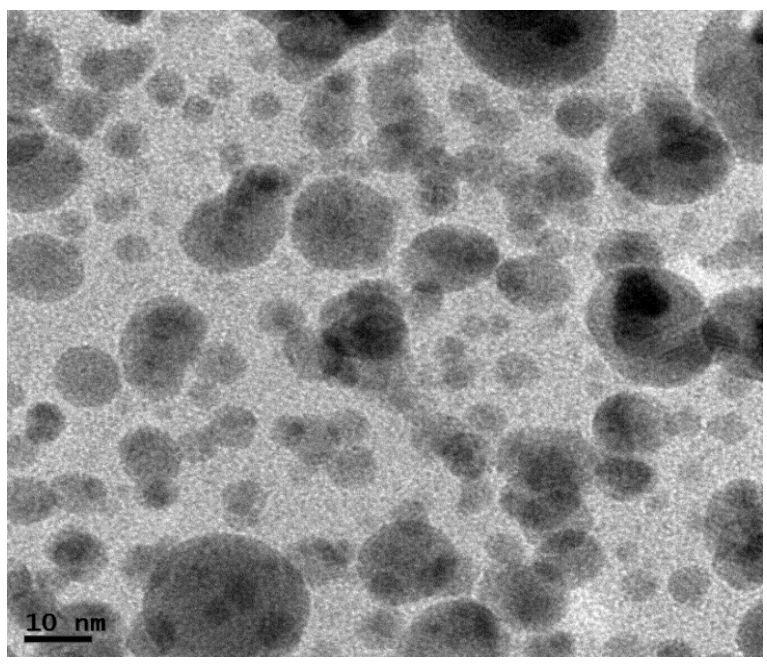

Figure.3. TEM image of Ag Nanoparticle

\section{FESEM and EDAX Analysis}

Fig. 4 shows the FESEM image of pure polyaniline and Pani/Ag nanocomposite. The FESEM shows that the samples of pure Pani have a submicron fibre and rod structure. However, in Pani/Ag nanocomposites, silver nanoparticles are well adhered on the Pani matrix and the silver nanoparticles are spherical in shape and covered with the layer of polyaniline. This could be attributed to strong affinity of silver to nitrogen of Pani and mechanism of silver migration and aggregation. Although some agglomerates are formed, it is also observed that the distribution of $\mathrm{Ag}$ nanoparticles is uniform in the polymer matrix and incorporation of $\mathrm{Ag}$ has a strong effect on the Pani's morphology. In Fig. 5, EDAX spectrometer analysis confirmed the presence of polymer components and silver nanoparticles in the nanocomposite.
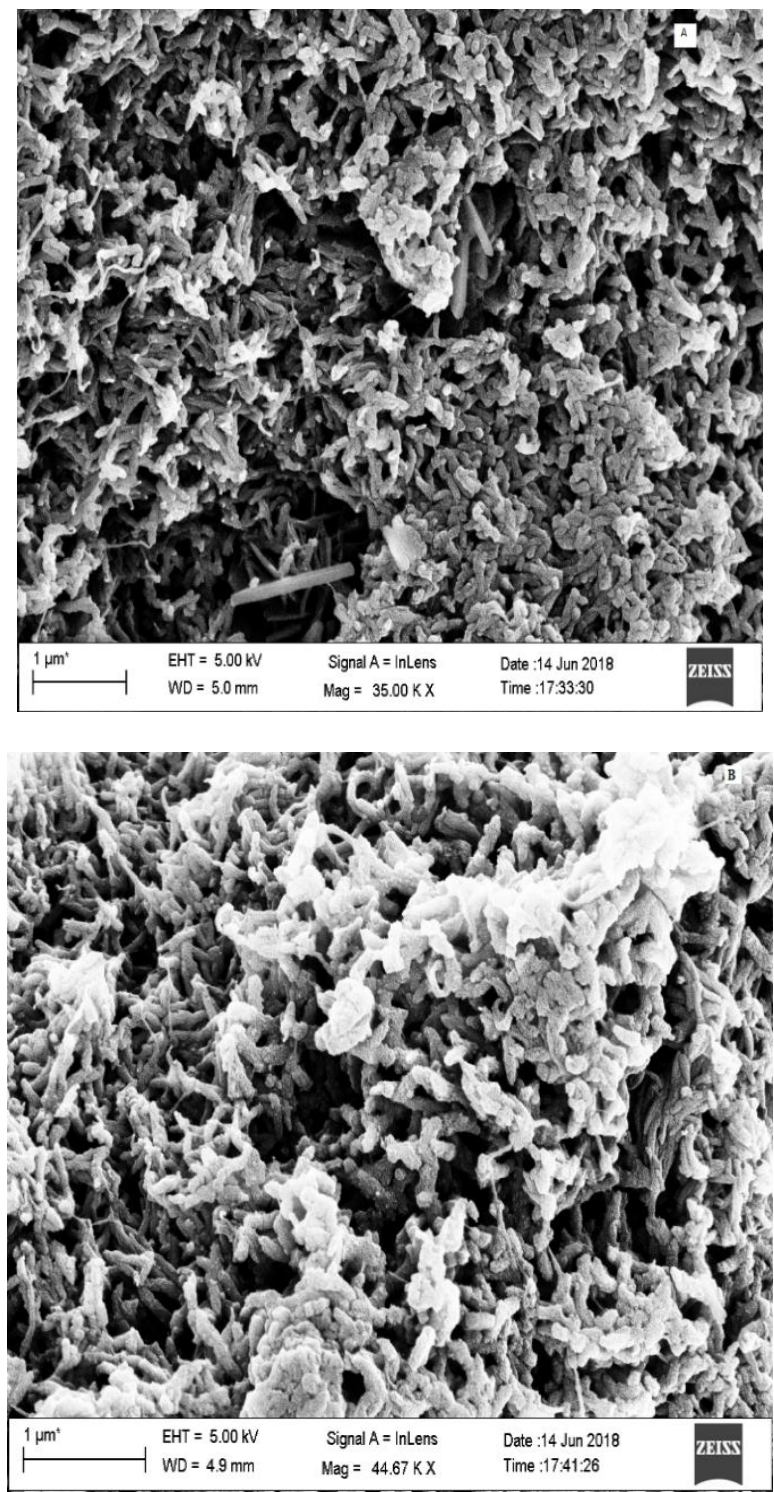


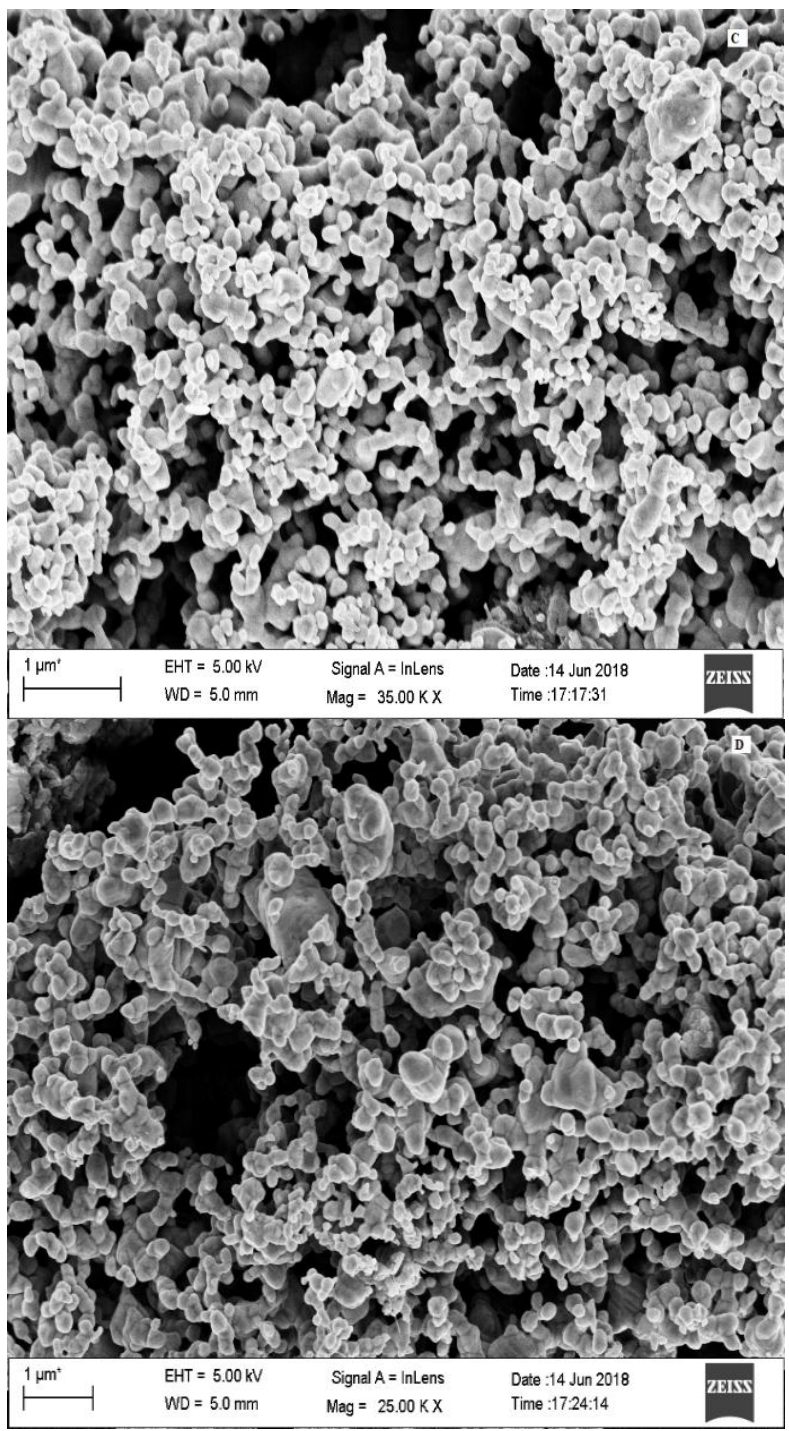

Figure 4. FESEM images of sample (A) Pure Pani and Pani/Ag nanocomposite prepared with $\mathrm{Ag}$ concentration of (B) $0.1 \mathrm{~mol} \%$, (C) $0.3 \mathrm{~mol} \%$ and (D) $0.5 \mathrm{~mol} \%$.

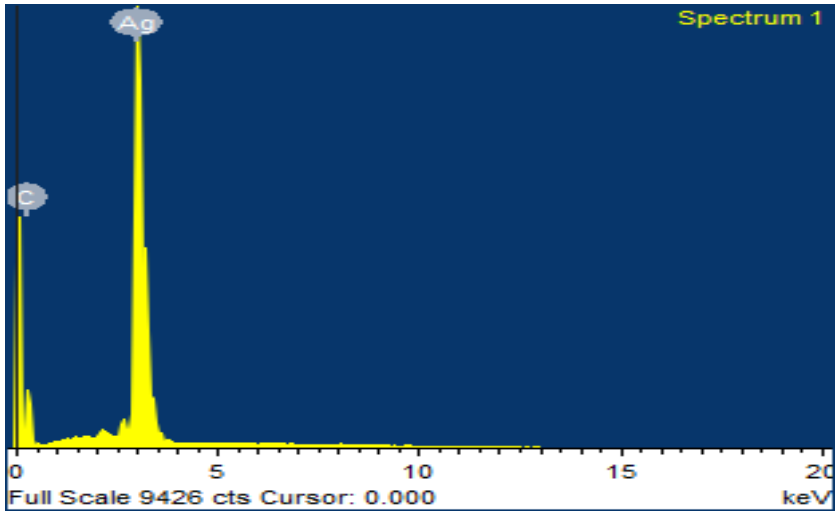

Figure 5. EDAX of Pani/Ag(0.5\% mol) nanocomposite

\section{UV-Vis. Study}

Figure 6 shows the absorption spectra of pure Pani and pani/Ag nanocomposite. The spectrum of pure PANI shows two characteristics absorption edge at the wavelength $\sim 298$ - $302 \mathrm{~nm}$ and $\sim 620-628 \mathrm{~nm}$. These absorption edges are attributed to the $\pi \rightarrow \pi^{*}$ transition of electrons in benzenoid ring present in the Pani structure and also due to exciton absorption of quinoid rings $n \rightarrow \pi^{*}$ [25-26]. In contrast to pure Pani, the Pani/Ag nanocomposites exhibit an additional absorption band between 400 and 425nm (Fig. 6). It corresponds to surface plasmon resonance absorption of the electrons in the conducting silver bands indicating that Ag nanoparticles are existed in the nanocomposite [27]. A similar plasmon peak of silver nanoparticle at around 425 $\mathrm{nm}$ was also reported for previous work on Pani/Ag nanocomposite [28].

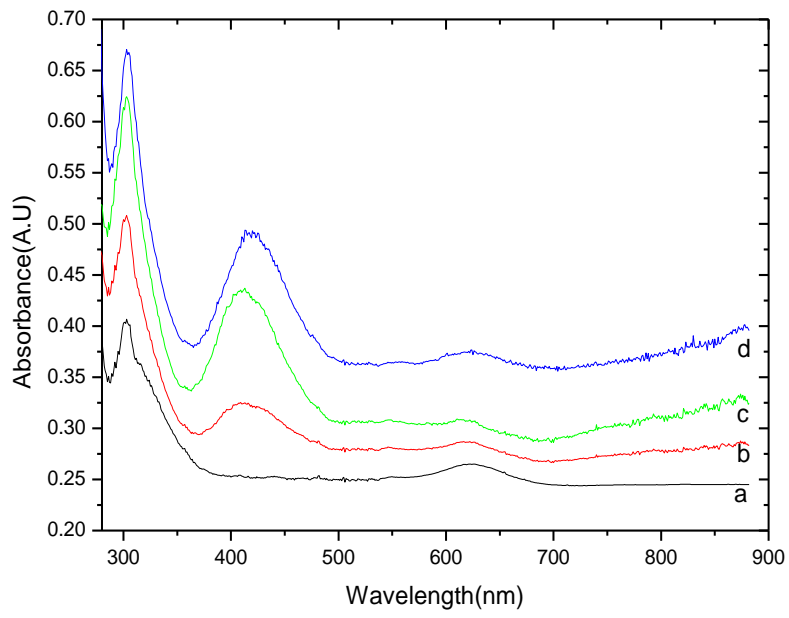

Figure 6. UV Vis spectrum of (a) Pure Pani and Pani/Ag nanocomposite prepared with $\mathrm{Ag}$ concentration of (b) 0.1 $\mathrm{mol} \%$, (c) $0.3 \mathrm{~mol} \%$ and (d) $0.5 \mathrm{~mol} \%$.

\section{E. Thermogravimetric Analysis}

The thermogravimetric analysis of pure pani and pani/Ag nanocomposite is shown in the Fig. 7. It is observed that the thermal decomposition of both pure Pani and Pani/Ag nanocomposite take place at three different stages of weight loss. The first weight loss below $200{ }^{0} \mathrm{C}$ is due to evaporation of adsorbed moisture or loss of other volatile components retained in the composite. The second weight loss from $200{ }^{0} \mathrm{C}$ to $330{ }^{0} \mathrm{C}$ can be attributed to the removal of higher oligomers of Pani [29]. The third and major weight loss after $\sim 350{ }^{0} \mathrm{C}$ is due to the structural decomposition of Pani resulting in various degradation of products [30]. In the case of Pani/Ag nanocomposites, the amount of weight loss is found to be lower than those of pure Pani and the residue is increased with increasing silver content in the nanocomposite. This result indicates that incorporation of 
silver nanoparticle could improve the thermal stability of the Pani and also retard the thermal decomposition of the nanocomposites. From the present result, we note that Pani/Ag $(0.5 \% \mathrm{~mol})$ showed a larger residual weight of $47 \%$ (weight loss 53\%) at $700{ }^{\circ} \mathrm{C}$ compared to pure Pani.

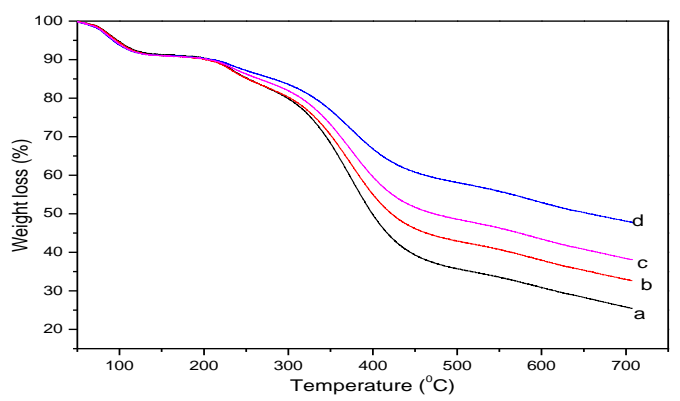

Figure 7. TGA curve of (a) Pure Pani and Pani/Ag nanocomposite prepared with Ag concentration of (b) 0.1 $\mathrm{mol} \%$, (c) $0.3 \mathrm{~mol} \%$ and (d) $0.5 \mathrm{~mol} \%$.

\section{F. DC Conductivity}

The variation of DC electrical conductivity of Pani/Ag nanocomposite with different molar concentration of silver is shown in the Fig. 8. The electrical conductivity of the Pani/Ag nanocomposite with $0.1 \%, 0.3 \%$ and $0.5 \% \mathrm{~mol}$ of $\mathrm{Ag}$ was found to be $0.81 \mathrm{~S} / \mathrm{cm}, 2.42 \mathrm{~S} / \mathrm{cm}$ and $3.61 \mathrm{~S} / \mathrm{cm}$ respectively, whereas that of the pure Pani was found to be $0.32 \mathrm{~S} / \mathrm{cm}$. This result shows that electrical conductivity of Pani significantly increased more than ten times after the incorporation of silver nanoparticles. This increase in conductivity may be due to enhancement in the mobility of charge carriers owing to increased tunnelling probability [31]. It is also believed that the incorporation of Ag into Pani forms a conducting bridge between the different Pani chains leading to an increase in electrical conductivity because both $\mathrm{Ag}$ and Pani are good conducting.

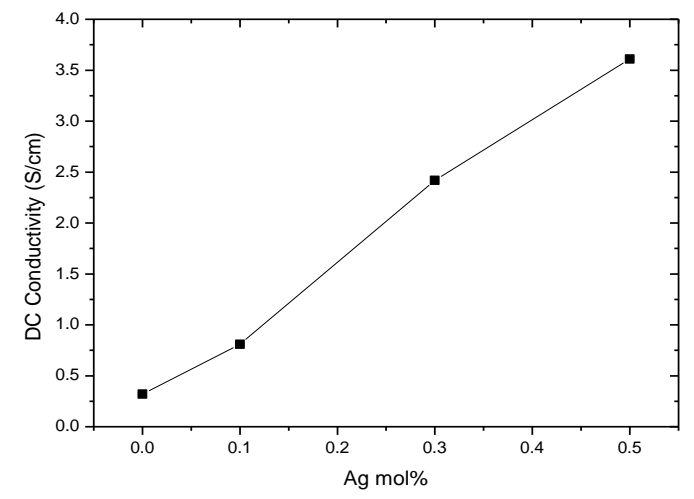

Figure 8. DC conductivity of Pani/Ag nanocomposite

\section{G. Gas sensing study}

Fig. 9 shows the electrical response of pure Pani and Pani/Ag $(0.5 \% \mathrm{~mol})$ nanocomposite sensor to 100,200 and
$300 \mathrm{ppm}$ of chloroform at room temperature. As observed from Fig. 9, for pure Pani the sensitivity value $\frac{\Delta R}{R_{\text {air }}}$ gradually increases upon exposure to chloroform but fail to return to its base line. The increase in sensitivity of pure Pani on exposure of chloroform is due to the interaction of chloroform molecules at dopant sites, viz. $N^{+}-H$ site created by $\mathrm{Cl}^{-}$. There is high probability that chloroform molecules mat attack dopant sites causing neutralizations of loosely bound couple formed with $\mathrm{Cl}^{-}$leading to appreciable increase in the resistance (and apparently $\frac{\Delta R}{R_{\text {air }}}$ value) of pure Pani [32]. On the contrary, Pani/Ag showed stable base line and improved sensing response for all chloroform concentration when compare to that of pure Pani. This significance increase in sensing response of Pani/Ag nanocomposite on exposure to chloroform can be attributed to the improved sensing mechanism of Pani and large degree of interactions between nanocomposite and vapours due to large surface area of Pani when it is coated over the $\mathrm{Ag}$ nanoparticles.
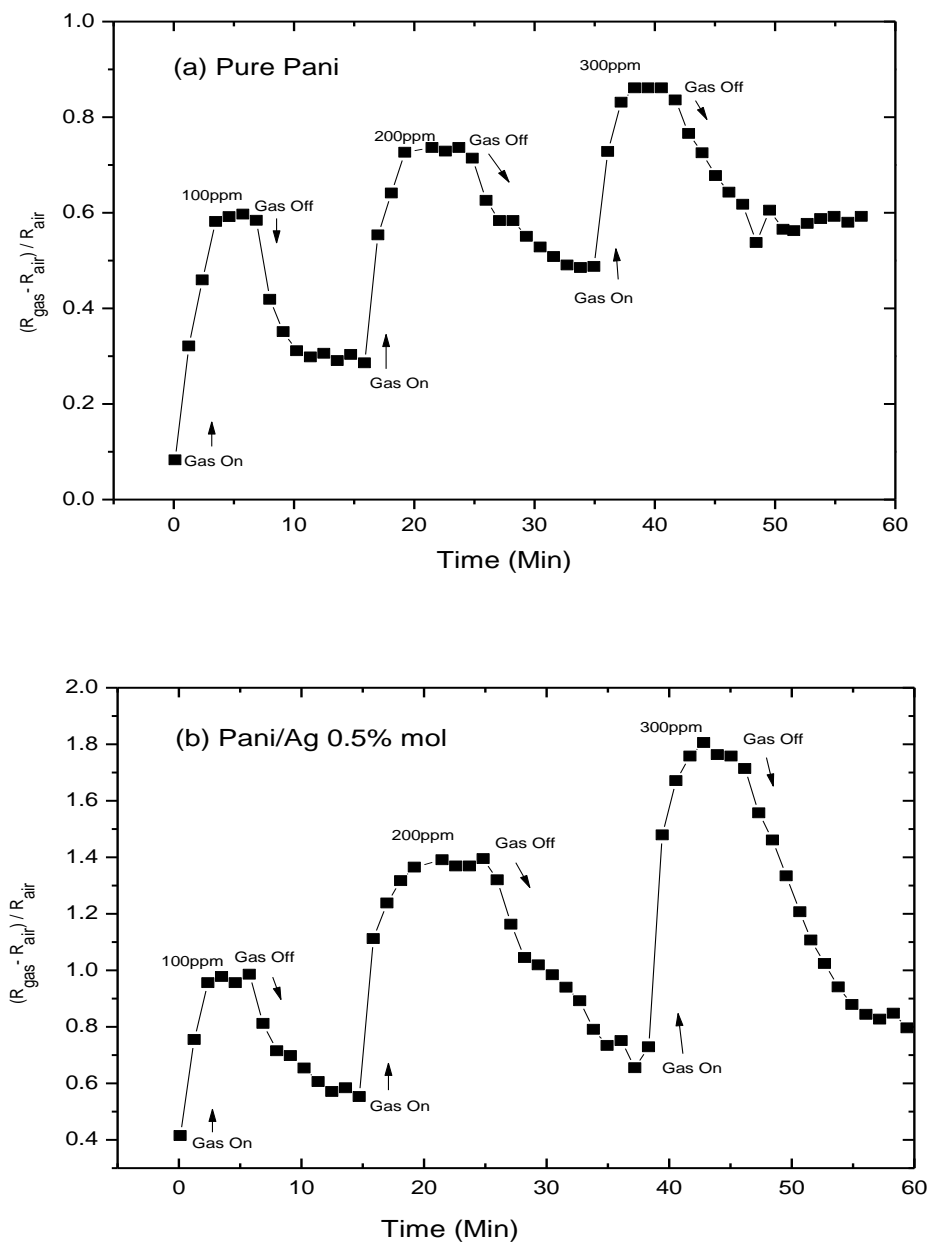

Figure 9. Sensing reproducibility of (a) pure Pani and (b)Pani with $0.5 \mathrm{~mol} \% \mathrm{Ag}$ to Chloroform 


\section{CONCLUSIONS}

In this paper, Polyaniline-Silver (Pani/Ag) nanocomposite were synthesis by an in-situ chemical polymerization method. The structural morphology, thermal stability, DC electrical conductivity and chloroform-sensing mechanism of the nanocomposite were examined using different techniques. Thermal stability of Polyaniline significantly improved after the incorporation of $\mathrm{Ag}$ nanoparticles to pure Polyaniline. The DC conductivity of Pani/Ag nanocomposite was recorded ten time higher than pure Pani. Pani/Ag nanocomposite showed better ammonia sensitivity and recovery for chloroform when compare with pure Pani.

\section{ACKNOWLEDGEMENTS}

The authors sincerely acknowledge UGC, India for the financial support. The author would like to acknowledge NEIST Jorhat, IIT Guwahati and Tezpur University for providing the necessary facilities for sample characterisation.

\section{REFERENCES}

[1]. Gordana, Ćirić-Marjanović, "Recent advances in polyaniline composites with metals, metalloids and nonmetals", Synthetic Metals, Vol 170, pp. 31- 56, 2013.

[2]. Manoj K. Sharma, M. K. Ambolikar, A. S.; Aggarwal, S. K, "In situ synthesis of gold-polyaniline composite in nanopores of polycarbonate membrane” J. Mater. Sci.,Vol.46, pp. 5715, 2011.

[3]. Naseri M, Fotouhi L, Ehsani A, "Recent Progress in the Development of Conducting Polymer-Based Nanocomposites for Electrochemical Biosensors Applications: A Mini-Review", Chem. Rec., Vol. 18, Issue 6, pp. 599-618, 2018.

[4]. A. Kitani, T. Akashi, K. Sugimoto, and S. Ito, "Electrocatalytic oxidation of methanol on platinum modified polyaniline electrodes" Synth. Met. Vol. 121, Issue 1-3, 1301, 2001.

[5]. M L Rozemarie, B Andrei, H Liliana, R Cramariuc, O Cramariuc, "Electrospun Based Polyaniline Sensors - A Review", IOP Conference Series: Materials Science and Engineering, Vol. 209, pp. 012063, 2017.

[6]. S Panday, "Highly sensitive and selective chemiresistor gas/vapor sensors based on polyaniline nanocomposite: A comprehensive review", Journal of Science: Advanced Materials and Devices, Vol. 1, pp. 431-453, 2016.

[7]. Y. Xia, K. Sun, J. Ouyang, "Solution-processed metallic conducting polymer films as transparent electrode of optoelectronic devices", Adv. Mater, Vol. 24, pp. 2436-2440, 2012.

[8]. Blinova, N. V.; Stejskal, J.; Trchov_a, M.; Sapurina, I.; CiricMarjanovic, G, "Polyaniline-silver composites prepared by the oxidation of aniline with silver nitrate in acetic acid solutions", Polym. Int, Vol. 59, pp. 437-446, 2010.

[9]. Sun, L.; Shi, Y.; Li, B.; Chu, L.; He, Z.; Liu, J, "Synthesis and characterization of polypyrrole/Au nanocomposites by microemulsion polymerization”, Colloids Surf. A, Vol. 397, pp. 811, 2012.

[10]. D K. Dutta, S. Das, D. Rana, P. P. Kundu, "Enhancements of Catalyst Distribution and Functioning Upon Utilization of Conducting Polymers as Supporting Matrices in DMFC: A Review”, Polym. Review, Vol. 55, Issue 1, pp. 1-56. 2015.

[11]. S. Z. Wu, F. Zeng, F. X. Li, and Y. L. Zhu, "Ammonia sensitivity of polyaniline films via emulsion polymerization", Eur. Polym. Journal, Vol. 36, Issue 4, pp. 679-683, 2000.

[12]. G. D. Khuspe, S. T. Navale, D. K. Bandgar, R. D. Sakhare, M. A. Chougule, and V. B. Patil, " $\mathrm{SnO}_{2}$ nanoparticles-modified polyaniline films as highly selective, sensitive, reproducible and stable ammonia sensors", Electron. Mater. Lett., Vol. 10, Issue 1, pp. 191-197, 2014.

[13]. Roopa J, Muniraj R, Magaraj T M, B S Satya Narayana, K S Geetha, "Development of a Conducting Polymer [polyaniline] based gas sensor", International Conference on Electrical, Electronics, Signals, Communication and Optimization (EESCO) (IEEE), 2015.

[14]. T.K. Sarma, D. Chowdhury, A. Paul, A. Chattopadhyay, "Synthesis of Au nanoparticle-conductive polyaniline composite using $\mathrm{H} 2 \mathrm{O} 2$ a oxidising as well as reducing agent", Chem. Commun., Vol.14 pp.1048-1049, 2002.

[15]. W. Xue, H. Qiu, K. Fang, J. Li, J. Zhao, M. Li, "Electrical properties of the composite pellets containing DBSA-doped polyaniline and Fe nanoparticles", Synth. Met., Vol. 156 833-837, 2006.

[16]. S. Sharma, C. Nirkhe, S. Pethkar, A.A. Athawale, "Chloroform vapour sensor based on copper/polyaniline nanocomposite", Sens. Actuators, B: Chem., Vol. 85, pp. 31-136, 2002.

[17]. Mudassir Hasan, Mohd Omaish Ansari, Moo Hwan Cho, Moonyong Lee, "Electrical Conductivity, Optical Property and Ammonia Sensing Studies on $\mathrm{HCl}$ Doped Au@Polyaniline Nanocomposites", Electron. Mater. Lett., Vol. 11, Issue 1 pp. 1-6, 2015.

[18]. A.A. Athawale, S.V. Bhagwat, P.P. Katre, "Nanocomposite of Pd-polyaniline as a selective methanol sensor", Sens. Actuators, B: Chem., Vol. 114, pp. 263-267, 2006.

[19]. Irma Zulayka Mohamad Ahada, Sulaiman Wadi Harunb, Seng Neon Gan, Sook Wai Phang, "Polyaniline (PAni) optical sensor in chloroform detection", Sensors and Actuators B, Vol. 261, pp. 97-105, 2018.

[20]. Lorraine Mulfinger, "Synthesis and Study of Silver Nanoparticles", Journal of Chemical Education, Vol. 84, Issue 2, pp. 322- 327, 2007.

[21]. F. M. Smits, "Measurement of Sheet Resistivities With the FourPoint Probe", The Bell System Technical Journal, pp. 711-718, 1958.

[22]. Qingming Jia, Shaoyun Shan, Lihong Jiang, Yaming Wang, "One-Step Synthesis of Polyaniline Nanofibers Decorated with Silver”, Appl. Plym. Sci., Vol. 115, 26-31, 2010.

[23]. K. Gupta, P. C. Jana and A. K. Meikap, "Optical and Electrical Transport Properties of Polyaniline-Silver Nanocomposite," Synthetic Metals, Vol. 160, Issue 13-14, pp. 1566-1573, 2010.

[24]. B.D. Cullity, Elements of X-ray Diffraction, M.A. Reading, Addison-Wesley, 1978. 
[25]. E. Erdem, M. Karakisla, M. Sacak, "The chemical synthesis of conductive polyaniline doped with dicarboxylic acids", Eur. Polym. J., Vol. 40, pp. 785-791, 2004.

[26]. D. Shihai, M. Hui, W. Zhang, "Fabrication of DBSA-doped polyaniline nanorods by interfacial polymerization", J. Appl. Polym. Sci., Vol. 109, pp.2842-2847, 2008.

[27]. Junhu Zhang et al, "Thin Films of Ag Nanoparticles Prepared from the Reduction of AgI Nanoparticles in Self-Assembled Films", Journal of Colloid and Interface Science, Vol. 255, pp. 115-118, 2002.

[28]. Arup Choudhury, "Polyaniline/silver nanocomposites: Dielectric properties and ethanol vapour sensitivity", Sensors and Actuators B, Vol. 138, pp. 318-325, 2009.

[29]. G. Chakraborty, S. Ghatak, A. K. Meikap, T. Woods, R. Babu, and W. J. Blau, "Characterization and electrical transport properties of polyaniline and multiwall carbon nanotube composites", J. Polym. Sci. Part B: Polym. Phy. Vol. 48, pp. 1767-1775, 2010.

[30]. M.O. Ansari, F. Mohammad, "Thermal stability, electrical conductivity and ammonia sensing studies on $p$-toluenesulfonic acid doped polyaniline:titanium dioxide ( $p$ TSA/Pani:TiO $\left.{ }_{2}\right)$ nanocomposites", Sens. Actuators B, Vol.157, pp. 122-129, 2011.

[31] M. Amrithesh, S. Aravind, S. Jayalekshmi, R.S. Jayasree, "Enhanced luminescence observed in polyanilinepolymethylmethacrylate composites", J. Alloys Compd., Vol. 449 pp. 176-179, 2008.

[32]. Satish Sharma, ChetanNirkhe, SushamaPethkar, Anjali A.Athawale, "chloroform vapour sensor based on copper/polyaniline nanocomposite", Senors and Actuators B, Vol. 85, Isuue 1-2, pp. 131-136, 2002. 